\title{
Endoplasmic Reticulum Stress-Mediated Apoptosis Involved in Indirect Recognition Pathway Blockade Induces Long-Term Heart Allograft Survival
}

\author{
Jianbin Xiang, ${ }^{1}$ Xiaodong Gu, ${ }^{1}$ Shiguang Qian, ${ }^{2}$ and Zongyou Chen ${ }^{1}$ \\ ${ }^{1}$ Department of General Surgery, Huashan Hospital, Fudan University, Shanghai 200040, China \\ ${ }^{2}$ Department of Immunology and General Surgery, Lerner Research Institute, Cleveland Clinic Foundation, Cleveland, \\ $\mathrm{OH} 44195$, USA
}

Correspondence should be addressed to Zongyou Chen, gxdgxd737@163.com

Received 13 December 2009; Accepted 24 February 2010

Academic Editor: Hanchun Yang

Copyright () 2010 Jianbin Xiang et al. This is an open access article distributed under the Creative Commons Attribution License, which permits unrestricted use, distribution, and reproduction in any medium, provided the original work is properly cited.

\begin{abstract}
Implementation of dendritic cell- (DC-) based therapies in organ transplantation can reduce dependency on nonspecific immunosuppression. Despite extensive research, mechanisms of equipped DCs inducing transplant tolerance remain incomplete. Here, we applied RNA interference technique to inhibit CD80 and CD86 expression in host bone marrow-derived DCs. This approach could specifically and effectively knock down CD80 and CD86 expression. T cells primed by these DCs inhibited allogeneic responses. Administration of recipient DCs loaded with alloantigen after CD80 and CD86 blockade prolonged cardiac allograft survival. We also found a higher percentage of apoptotic T cells in lymph tissues and grafts than that detected in control group. In addition, these T cells expressed high expression of GRP78 than controls, indicating activation of unfolded protein responses. Upregulation of CHOP expression among these cells suggested that the endoplasmic reticulum stress (ERS) response switched to a proapoptotic response. Our results indicated that ERS-induced apoptosis may be involved in allogeneic T-cell apoptosis, and the ERS-mediated apoptosis pathway may be a novel target in clinical prevention and therapy of allograft rejection.
\end{abstract}

\section{Introduction}

Dendritic cells (DCs) have been found to be the pivotal antigen presenting cells (APCs) in regulation of immune response [1]. Activation through the T cell receptor in the absence of costimulation is proposed to render responder $\mathrm{T}$ cells anergic or tolerant [2]. The costimulatory signal is delivered through interactions between the $\mathrm{T}$ cells and APCs and results from ligation of molecules such as CD28 and CD154 (CD40L), expressed on the $\mathrm{T}$ cells with their ligands CD80/CD86 and CD40 respectively, on APCs. Costimulation blockade targeting CD80/CD86 on DCs efficiently prevents acute heart or kidney rejection in many mouse and rat models [3-7]. First clinical trial has been conducted to assess co-stimulation blockade strategy in renal transplantation [8]. It may allow patients to avoid the adverse effects of calcineurin inhibitors, whilst providing equally effective immunosuppression.
There are direct and indirect pathways of allorecognition, and both of which have been postulated to have roles in allograft immunity [9]. Direct alloantigen (Ag) presentation, mediated by donor APCs, leads to vigorous $\mathrm{T}$ cell proliferation and is mainly involved in acute rejection [10]. As donor DCs undergo attrition, their role as presenters of alloAg subsides. Then recipient DCs that can infiltrate to the graft become the predominant APCs, and they present alloAg indirectly to $\mathrm{T}$ cells. This indirect pathway is closely related to chronic rejection [11]. However, more data showed that indirect recognition might play a more important role in whole allograft rejection [12-14] and indirect recognition also can initiate rapid skin graft rejection [15]. In addition, DCs cannot be obtained from deceased donors; so recipient DCs would potentially be taken into account in clinical transplantation.

RNA interference (RNAi) is a recently identified phenomenon in which small interference RNA (siRNA) interacts 
TABLE 1: Sequences of shRNA fragment for inserting lentivirus vector.

\begin{tabular}{lcccc}
\hline Name & $5^{\prime}$ & Sense & Loop & Antisense \\
\hline CD80 forward & $\mathrm{t}$ & aaggaaagaggaacgtatgaa & ttcaagaga & ttcatacgttcctctttcctt \\
CD80 reverse & tcgagaaaaaa & aaggaaagaggaacgtatgaa & tctcttgaa & ttcatacgttcctctttcctt \\
CD86 forward & $\mathrm{t}$ & gcacagagaaacttgatagtgt & ttcaagaga & acactatcaagtttctctgtgc \\
CD86 reverse & tcgagaaaaaa & gcacagagaaacttgatagtgt & tctcttgaa & acactatcaagtttctctgtgc \\
Scrambled forward & $\mathrm{t}$ & ttctccgaacgtgtcacgt & ttcaagaga & acgtgacacgttcggagaa \\
Scrambled reverse & tcgagaaaaaa & ttctccgaacgtgtcacgt & tctcttgaa & acgtgacacgttcggagaa \\
\hline
\end{tabular}

The bold characters represent the loop sequences and the italic characters represent the terminator sequences.

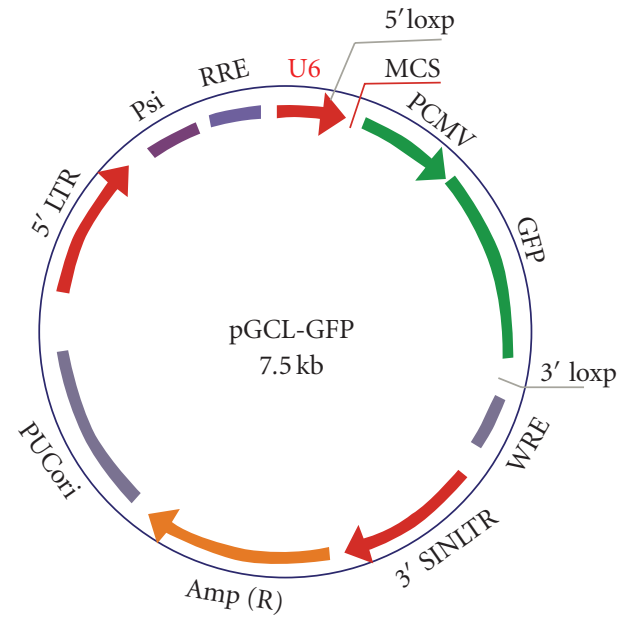

(a)

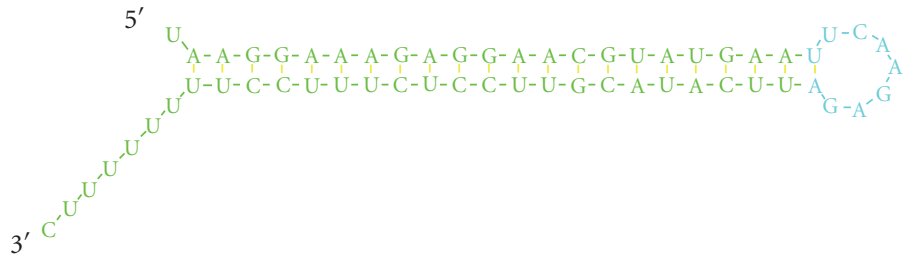

(b)

FIGURE 1: (a) Schematic presentation of the plasmid pGCL-GFP, which encodes an HIV-derived lentiviral vector containing a multiple cloning site (MCS) for insertion of shRNA constructs to be driven by an upstream U6 promoter and a downstream cytomegalovirus promoter-GFP fluorescent protein (marker gene) cassette flanked by loxp sites. (b) The stem-loop-encoded shRNA has sequence identity to a 21-nt region of CD80 mRNA.

with mRNA containing homologous sequences, and ultimately this interaction results in degradation of the target mRNA. Hill et al. [16] reported that transfection of DCs with siRNA specific for IL-12 p35 gene resulted in potent suppression of gene expression and blockade of bioactive IL-12 p70 production. This demonstrates that RNAi is a potential and useful tool to modulate DCs. Based on the attractive methodology of RNAi for silencing a particular gene expression, we used lentivirus mediated RNAi to suppress CD80 and CD86 expression on host DCs.

Endoplasmic reticulum is the organelle in which newly synthesized secretory and transmembrane proteins form their proper tertiary structure by posttranslational modification, folding, and oligomerization. However, many of these proteins are unfolded or misfolded by extracellular or intracellular stimuli. The accumulation of misfolded proteins constitutes a risk for living cells. Eukaryotic cells possess several mechanisms to adapt to endoplasmic reticulum stress (ERS) and thereby survive. If the cells are exposed to prolonged or strong ERS, the cells are destroyed by apoptosis. Recent evidence indicates that ERS signaling pathways play an important role in the pathogenesis of neurodegenerative disorders and diabetes [17]. Increasing evidences suggest ERS is involved in allograft injury [18].

At present, it is not known whether ERS is involved in peripheral tolerance. In this study, we detected that these DCs-pulsed alloAg could elicit lower proliferative responses and prolong heart allograft survival. Meanwhile, we characterized $\mathrm{T}$ cell apoptosis in vivo. For the first time, our study demonstrates that ERS-mediated apoptosis signal pathway is involved in $\mathrm{T}$ cell apoptosis after indirect recognition pathway blockade.

\section{Materials and Methods}

2.1. Animals. $\mathrm{C} 3 \mathrm{H} / \mathrm{HeJ}\left(\mathrm{C} 3 \mathrm{H} ; \mathrm{H}-2 \mathrm{~K}^{k}\right), \mathrm{C} 57 \mathrm{BL} / 6$ (B6; H$\left.2 \mathrm{~K}^{b}\right)$, and $\mathrm{BALB} / \mathrm{c}\left(\mathrm{H}-2 \mathrm{~K}^{d}\right)$ mice were purchased from Shanghai Laboratory Animal Center of Chinese Academy of Sciences (Shanghai, China) and were maintained in pathogen-free facility at Fudan University (Shanghai, China). Animals were fed with standard chow ad libitum and were used at 7-9 weeks of age. The animal experimental protocols were in accordance with Chinese Administration Rule of Laboratory Animal. 
TABLE 2: Sequences of primers.

\begin{tabular}{lcc}
\hline Gene name & Forward & Reverse \\
\hline Fas & ATGCATGACAGCATCCAAGA & TGCTGGCAAAGAGAACACAC \\
FasL & GCAGAAGGAACTGGCAGAAC & GCTGGTTGTTGCAAGACTGA \\
Bcl-xL & TTGCTGATGGCAACTTCAAC & CTCAGCCCATCTTCTTCCAG \\
GRP78 & GGTGAGTCGGATTGCAAGTT & TGTCTGGTCACTTCCGACTG \\
CHOP & ACCTGGGTGGGGAAGACTTT & TCTTCAAATTTGGCCCGAGT \\
$\beta$-action & TATCTCATCCCCAGGAAACG & CTGCTCCTTCTCCTTCATGC \\
\hline
\end{tabular}

2.2. Generation of Bone Marrow-Derived DCs. The BMderived myeloid DCs were propagated as described [19]. Briefly, the BM cells were removed from femurs and tibias of $\mathrm{C} 3 \mathrm{H}$ mice and depleted of erythrocytes by hypotonic lysis. The cells were cultured in 24-well plates $\left(1 \times 10^{6} /\right.$ well $)$ in $1 \mathrm{~mL}$ RPMI 1640 (Gibco, Gaithersburg, MD) supplemented with $10 \% \mathrm{v} / \mathrm{v}$ fetal bovine serum (FBS) and $10 \mathrm{ng} / \mathrm{mL}$ recombinant GM-CSF (R\&D Systems, Minneapolis, MN). All cultures were incubated at $37^{\circ} \mathrm{C}$ in $5 \%$ humidified $\mathrm{CO}_{2}$. Nonadherent granulocytes were removed after 48 hours of culture. Half media exchange was performed once every 48 hours. After 6 days of culture, $1 \mu \mathrm{g} / \mathrm{mL}$ LPS (Sigma, St. Louis, $\mathrm{MO}$ ) was added to the culture for 18 hours to allow for maturation. The purity of DC preparations was routinely monitored by flow cytometric analysis using anti-CD11c monoclonal antibody (mAb) (eBioscience, San Diego, CA). This DC preparation protocol could enrich CD11c+ cells more than $85 \%$.

2.3. Production of Recombinant Lentivirus. The recombinant lentivirus was constructed as previously described [20], with some modifications. In brief, short hairpin RNA (shRNA) fragments (Table 1) were hybridized with synthesized sense and antisense oligonucleotides. The hybridized CD80 shRNA fragment was cloned into the plasmid pGCL-GFP (Figure 1). Recombinant lentiviruses were produced by the transduction of $293 \mathrm{~T}$ cells. The $293 \mathrm{~T}$ cells were cotransduced with $20 \mu \mathrm{g}$ of pGCL-GFP, $15 \mu \mathrm{g}$ of pHelper1.0, and $10 \mu \mathrm{g}$ of pHelper2.0 by $100 \mu \mathrm{L}$ of Lipofectamine 2000 (Invitrogen, Garlsbad, CA). This recombinant lentivirus was designated CD80 lenti. The titers of CD80 lenti were determined with a green fluorescent protein (GFP) assay. CD86 lenti and NC lenti were constructed by a similar process. NC lenti contained scrambled shRNA fragment, an irrelevant shRNA with random nucleotides, and a GC ratio close to abovementioned shRNA.

2.4. Recombinant Lentivirus Transduction in DCs. Lentivirus transduction was conducted when DCs were 30\%-50\% confluent, lentivirus was added at different MOIs in serumfree medium at $37^{\circ} \mathrm{C}$ in $5 \%$ humidified $\mathrm{CO}_{2}$. After 4 hours, complete medium was added to the cells, and there was complete replacement of medium after 48 hours. Then, 4 days post transduction, marker gene expression was examined using fluorescent microscopy.
2.5. Ag Uptake. On day 6 of DCs culture, allogeneic splenocyte lysates were added to the culture at a DC: splenocyte equivalent ratio of $1: 10$ for 18 hours at $37^{\circ} \mathrm{C}$ in $5 \%$ humidified $\mathrm{CO}_{2}$. $\mathrm{B} 6$ splenocyte lysates were obtained by six cycles of freeze/thaw exposure in PBS.

2.6. Isolation of Graft-Infiltrating Lymphocytes. Grafts were harvested, minced, and homogenized with 5\% collagenase type II (Worthington, Lakewood, NJ) for 30 minutes at $37^{\circ} \mathrm{C}$. Tissue debris was removed by $100 \mu \mathrm{m}$ cell strainer. Lymphocytes were purified by lymphocyte separation medium (Mediatech, Herndon, VA) and then subjected to flow cytometry.

2.7. Flow Cytometric Analysis. Expression of cell surface Ag was detected on a FACScan (BD Biosciences, San Jose, CA) and analyzed using CellQuest software (BD Biosciences). Cells were stained with the following mAb: PE-IAk, PECD40, PE-CD80, PE-CD86 (eBioscience), and FITC-CD3 (BD Biosciences). Isotype-matched irrelevant mAbs were used as negative controls. Apoptosis was assessed by PEAnnexin V staining (BD Biosciences).

2.8. Primary and Secondary Mixed Lymphocyte Reaction (MLR). For primary MLR, nylon wool-eluted spleen $\mathrm{T}$ cells $\left(2 \times 10^{5}\right)$ from $\mathrm{C} 3 \mathrm{H}$ mice were used as responders. $\gamma$ irradiated $(20 \mathrm{~Gy})$ DCs derived from C3H BM were used as stimulators. For secondary MLR, $3 \times 10^{6} \mathrm{DCs}$ were injected into $\mathrm{C} 3 \mathrm{H}$ mice. Animals were sacrificed 7 days after injection. Isolated splenic $\mathrm{T}$ cells from DCs-injected $\mathrm{C} 3 \mathrm{H}$ mice were responders and restimulated with $\gamma$-irradiated $\mathrm{B} 6$, $\mathrm{C} 3 \mathrm{H}, \mathrm{BALB} / \mathrm{c}$ Ag-pulsed C3H-derived DCs. Cultures were maintained in complete medium for 3 days at $37^{\circ} \mathrm{C}$ in $5 \%$ humidified $\mathrm{CO}_{2}$. $\left[{ }^{3} \mathrm{H}\right]$-TdR $(0.5 \mu \mathrm{Ci} /$ well $)$ was added for the final 18 hours of culture. Cells were harvested onto glass fiber disks using an automated system, and incorporation of $\left[{ }^{3} \mathrm{H}\right]$-TdR into DNA was assessed by Wallac 1450 liquid scintillation counter (PerkinElmer, Boston, MA). Results were expressed as mean counts per minute $(\mathrm{cpm}) \pm 1 \mathrm{SD}$.

2.9. Heterotopic Heart Transplantation. Cervical vascularized heart transplantation was performed from B6 donors to size-matched $\mathrm{C} 3 \mathrm{H}$ recipients using a cuff technique. Graft survival was assessed by daily palpation. The operation 
was successful when grafts continued to beat for more than 3 days. Rejection was defined as total cessation of cardiac contraction. To assess the effect of equipped DCs on allograft survival, animals received $2 \times 10^{6}$ cells administered intravenously through the lateral tail vein 7 days prior to heart transplantation in the absence of immunosuppression.

2.10. Semiquantitative RT-PCR. Total RNA of splenic T cells was extracted using Trizol reagent according to the manufacturer's instructions (Invitrogen). RNA was then reverse-transcribed into $\mathrm{CDNA}$, using random primers and Superscript II reverse transcriptase (Invitrogen). For semiquantitative RT-PCR, the PCR amplification was performed using Taq DNA polymerase (Invitrogen). PCR products were analyzed on agarose gels stained with ethidium bromide and photographed. The primers of each target gene were shown in Table 2.

2.11. Statistical Analysis. Statistical analysis was performed with Stata 8.0 software (Stata, College Station, TX). The data was given as mean \pm 1 SD. Statistical comparisons between groups were preformed using a one-way ANOVA followed by a Scheff's test, as appropriate. Graft survival between groups of transplanted animals was compared using the log-rank test for comparison of survival curves. Values of $P<.05$ were considered statistically significant.

\section{Results}

3.1. Lentivirus Mediates Efficient RNAi in DCs. To determine the lentiviral transduction efficiency in DCs, GFP expression was examined by microscopy and flow cytometry at different MOIs on day 4 after transduction. Lentivirus demonstrated a high $(79.8 \% \pm 5.0 \%)$ transduction efficiency in DCs at MOI of 20 (Figure 2). The percentage of GFP-expressing cells remained minor changes when MOI is $>20$. Therefore, in the following experiments all viral titers were at MOI of 20. To blockade both CD80 and CD86 expression, DCs were transduced with both CD80 lenti and CD86 lenti. DCs were collected after LPS stimulation. As measured by flow cytometry, we found that CD80 lenti and CD86 lenti together could enhance suppressive effect on CD80 and CD86 expression (Figure 3(a)). After the activation of LPS, untreated DCs underwent marked maturation progress. But as to those transduced DCs LPS failed to bring great changes of the CD80 and CD86 expression. On the other hand, CD80 lenti and CD86 lenti had no effect on expression of unrelated proteins (such as MHC-II and CD40) (Figure 3(b)). Our study indicates that recombinant lentivirus can provide highly efficient and specific CD80 and CD86 knockdown in DCs.

3.2. DCs after CD80 and CD86 Blockade Induces T Cell Specific Hyporesponsiveness. To explore the influence of these DCs on $\mathrm{T}$ cell response, DCs were transduced with recombinant lentivirus. After pulsing B6 splenocyte lysates, DCs were used as stimulators of naïve $\mathrm{C} 3 \mathrm{H}$ splenic T cells. CD80 lenti and CD86 lenti-transduced DCs were poor stimulators of T cells
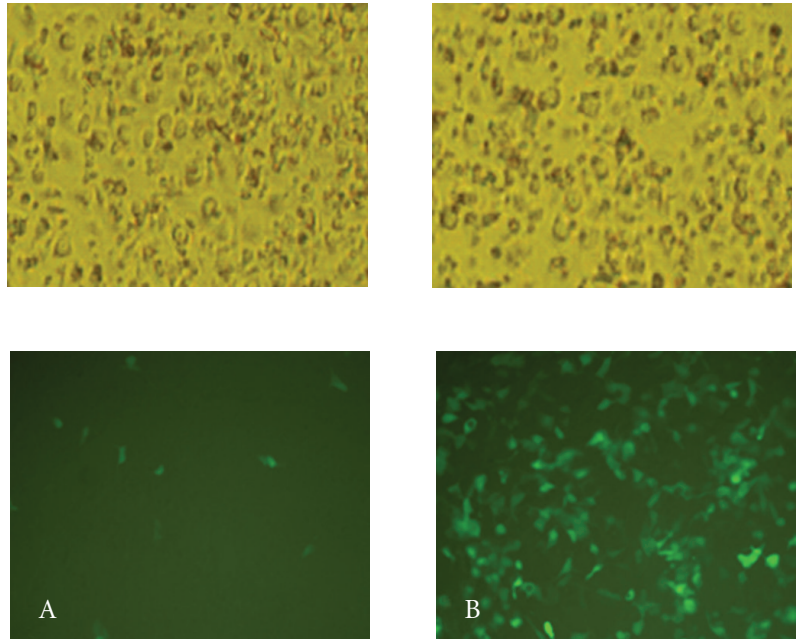

(a)

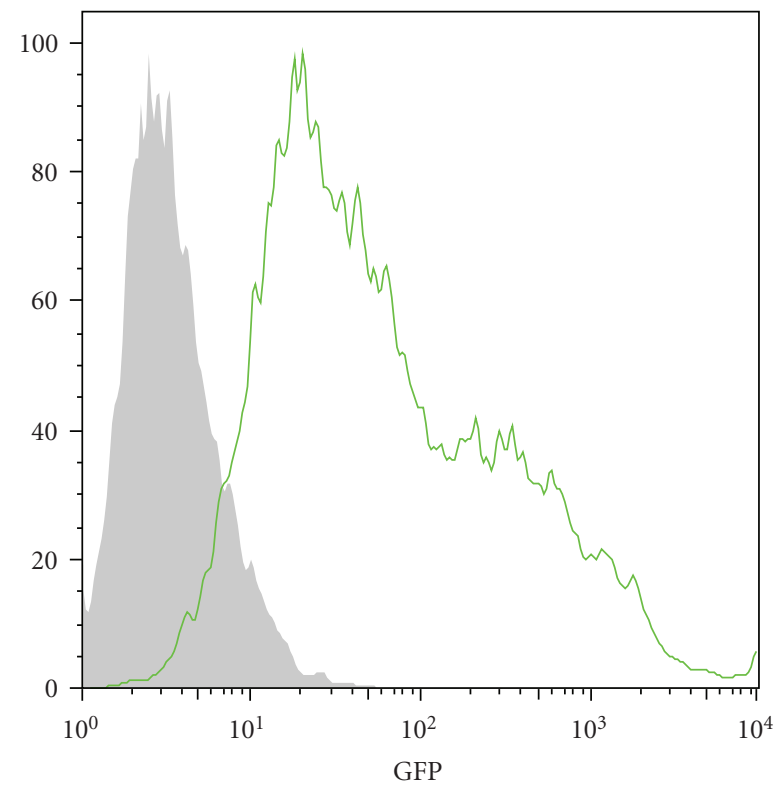

(b)

Figure 2: Transduction efficiency was estimated 4 days after transduction at indicated MOIs. (a) GFP expression was observed under light microscopy (top) or fluorescence microscopy (bottom). (A) MOI of $5(\times 100)$. (B) MOI of $20(\times 100)$. DCs saw an increase in expression of GFP that peaked when MOI $=20$. (b) The efficiency of infection was determined by assaying GFP expression by flow cytometry (green line) and comparing with uninfected control cells (grey peak).

compared to NC lenti-transduced DCs or no treated DCs (Figure 4(a)). Next, these DCs were injected into C3H mice through the lateral tail vein. As shown in Figure 4(b), T cells from $\mathrm{C} 3 \mathrm{H}$ mice primed by injection of $\mathrm{CD} 80$ lenti and $C D 86$ lenti-transduced DCs exhibited marked reduced responses to alloAg (B6) versus to third party unrelated $\mathrm{Ag}(\mathrm{BALB} / \mathrm{c})$ pulsed $\mathrm{C} 3 \mathrm{H}$-derived DCs. In contrast, $\mathrm{T}$ cells from $\mathrm{C} 3 \mathrm{H}$ mice primed by injection of NC lenti-transduced or no treated DCs exhibited markedly increased proliferative responses 


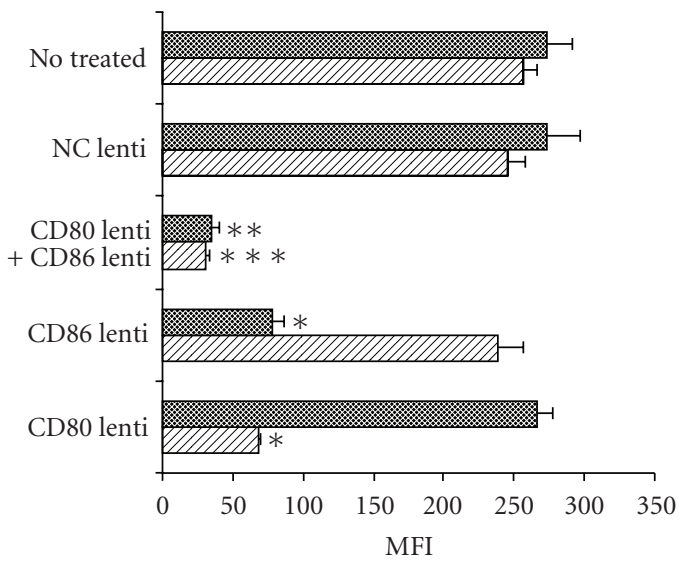

的86

$\square \mathrm{CD} 80$

(a)

No treated

LPS
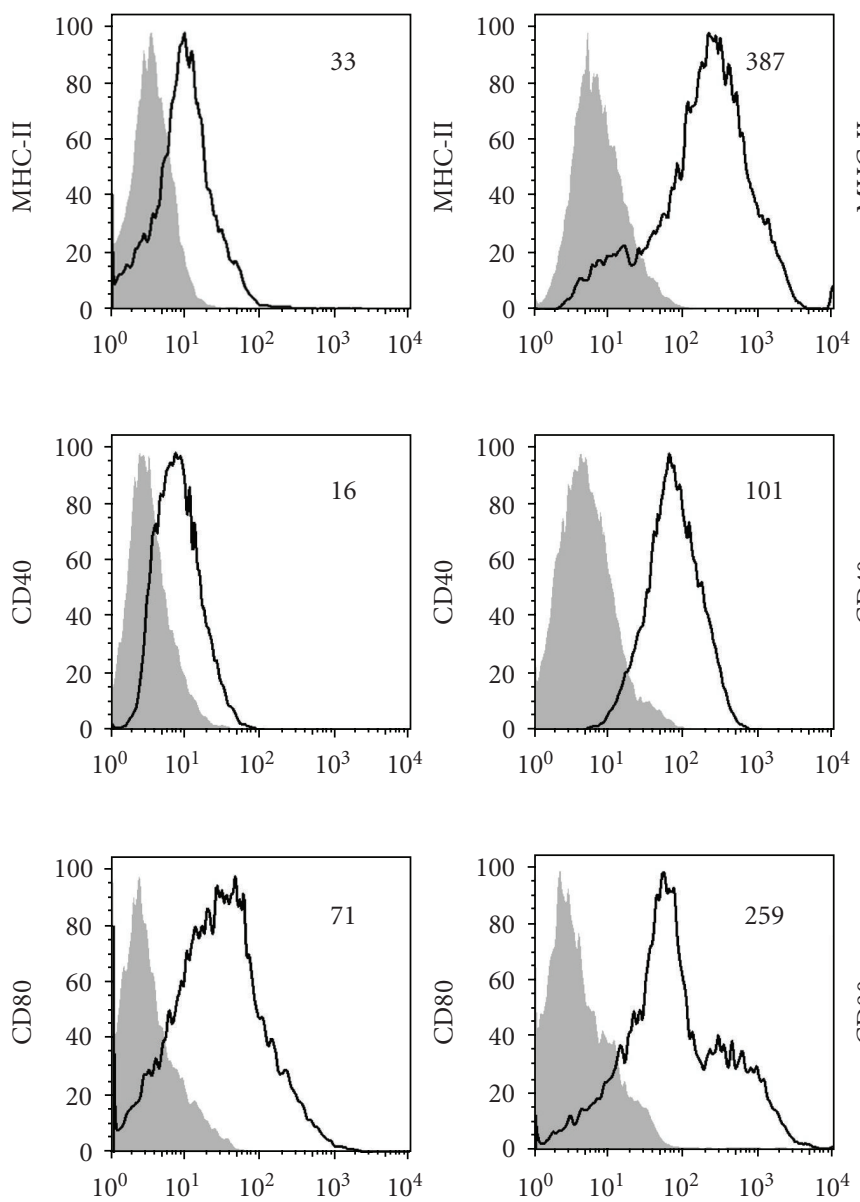

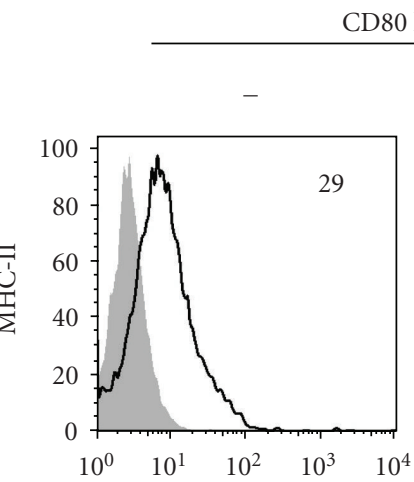

CD80 lenti + CD86 lenti
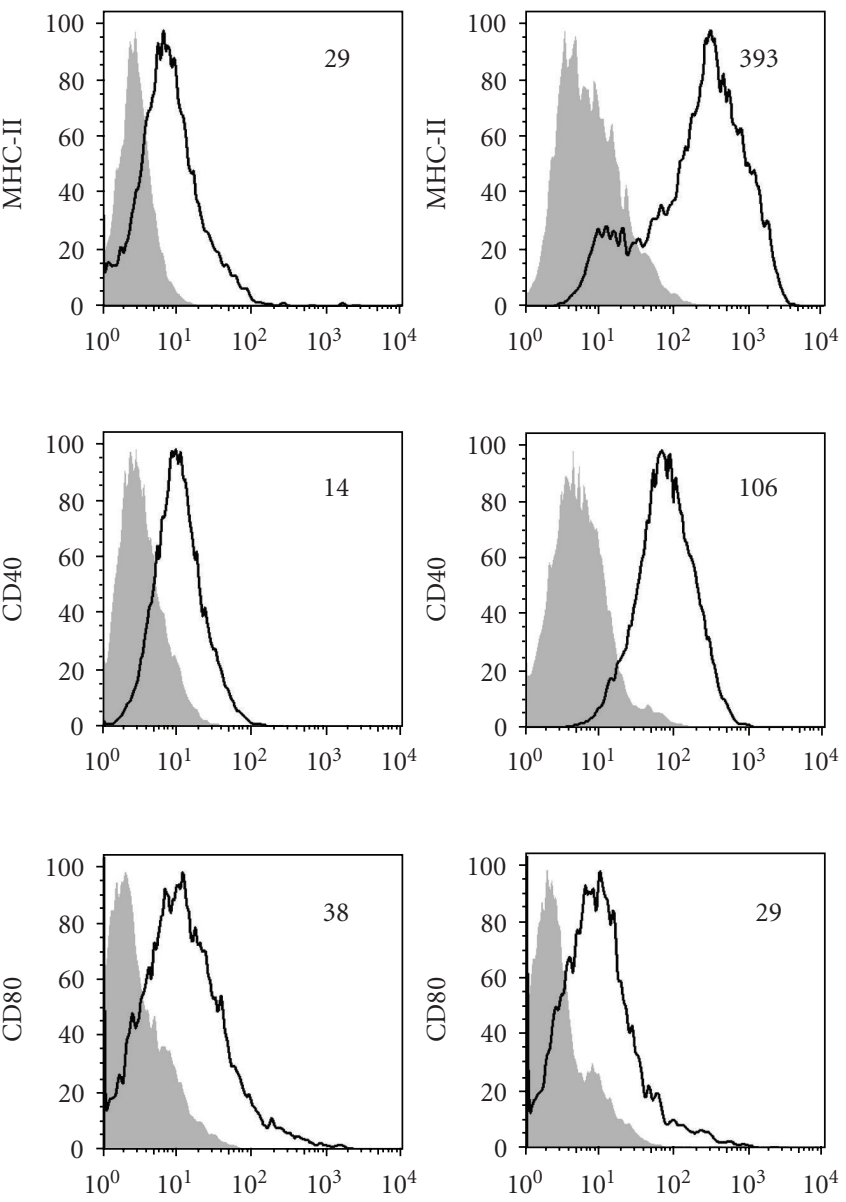

(b)

FIgUre 3: Continued. 

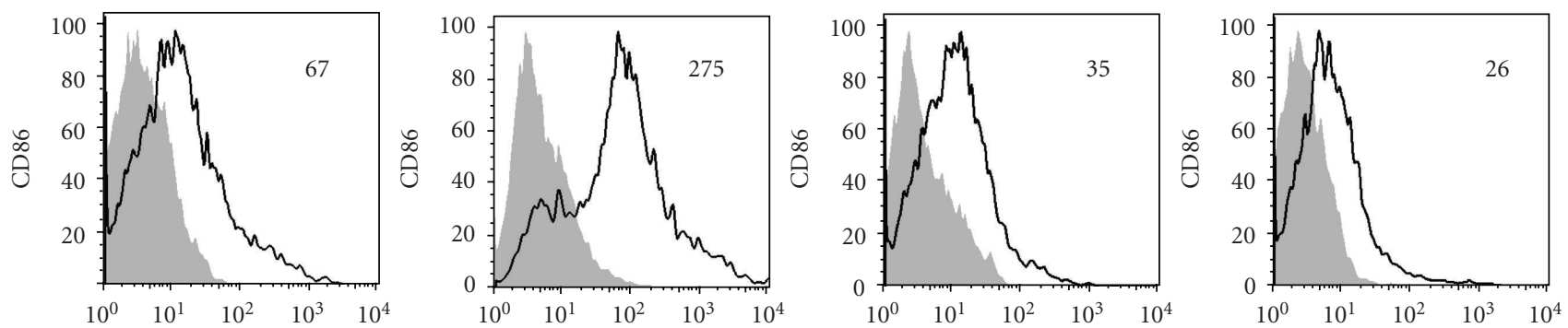

(b)

Figure 3: DCs were transduced with nothing (No treated), CD80 lenti, CD86 lenti, NC lenti, or both CD80 lenti and CD86 lenti. (a) After 6 days of culture, the cells were then incubated with LPS $(1 \mu \mathrm{g} / \mathrm{mL})$ for 18 hours. Expression of CD80 and CD86 was determined by flow cytometry. The data show the effect of recombinant lentivirus on inhibition of target molecule expression. Values expressed as mean $\pm 1 \mathrm{SD}$ from three independent experiments; ${ }^{*} P<.05$ compared to $N C$ lenti/No treated; ${ }^{* *} P<.05$ compared to CD80 lenti; $* * * P<.05$ compared to CD86 lenti. (b) C3H BM-derived DCs were cultured over 6 days. DCs were stimulated or unstimulated with LPS (1 $1 \mathrm{~g} / \mathrm{mL})$ for 18 hours. Upregulation of CD80 and CD86 in response to LPS was also suppressed by CD80 lenti and CD86 lenti. The mean fluorescence intensity (MFI) of GFP+ cells expressing the marker of interest is indicated.

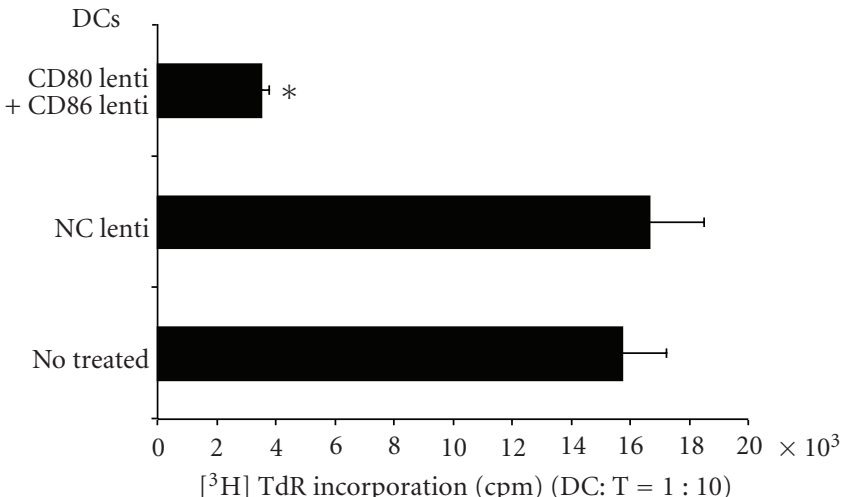

(a)

$\mathrm{Ag}$
$\mathrm{BALB} / \mathrm{c}$
$\mathrm{C} 3 \mathrm{H}$
$\mathrm{B} 6$
$\mathrm{BALB} / \mathrm{c}$
$\mathrm{C} 3 \mathrm{H}$
$\mathrm{B} 6$
$\mathrm{BalB} / \mathrm{c}$
$\mathrm{C} 3 \mathrm{H}$
$\mathrm{B} 6$

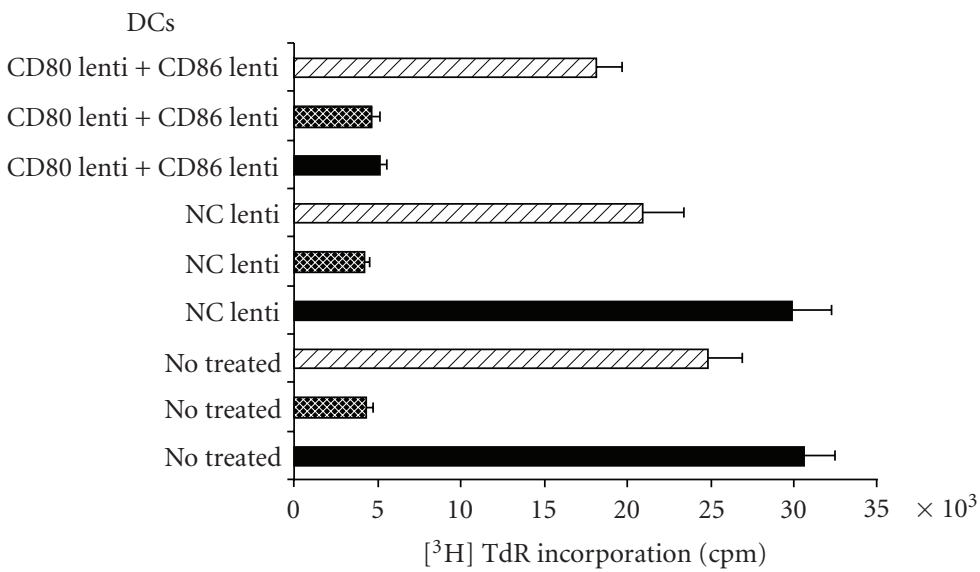

C3H DCs pulsed with Ag: C3H spleen T cells $=1: 10$

(b)

Figure 4: (a) B6 alloAg-pulsed, CD80 lenti- and CD86 lenti-transduced DCs were inferior stimulators of naïve syngeneic C3H T cells, compared to alloAg-pulsed NC lenti-transduced DCs or no treated DCs. ${ }^{*} P<.05$ compared to NC lenti/No treated. (b) DCs loaded with debris $\mathrm{B} 6$ spleen cells were injected into syngeneic $\mathrm{C} 3 \mathrm{H}$ mice $\left(3 \times 10^{6} / \mathrm{mouse}\right)$. Seven days later, recipients were sacrificed and splenic T cells challenged ex vivo with $\gamma$-irradiated C3H DCs pulsed with Ag (BALB/c, C3H, or B6 splenocyte lysates). Data are expressed as cpm \pm 1 SD from triplicate cultures and are representative of four separate experiments. 


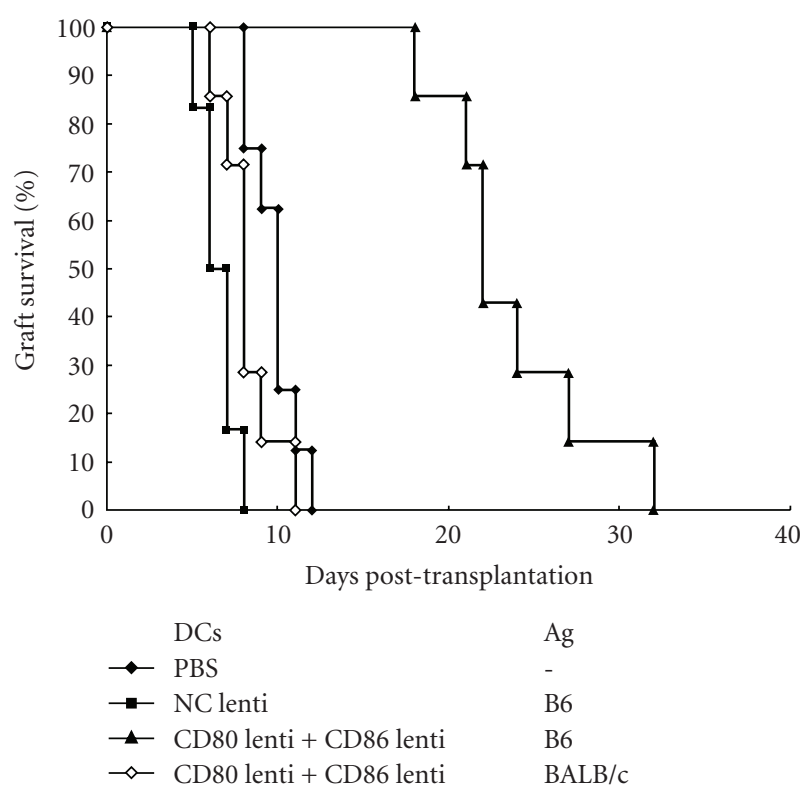

FIgure 5: AlloAg-pulsed DCs in NC lenti group, or CD80 lenti and CD86 lenti group, and third party Ag-pulsed DCs in CD80 lenti and CD86 lenti group were injected into $\mathrm{C} 3 \mathrm{H}$ mice, 7 days before the transplantation with B6 heart grafts. ${ }^{*} P<.05$ compared to other groups.

to donor alloAg and third party Ag-pulsed C3H-derived DCs. However, $\mathrm{T}$ cells from $\mathrm{C} 3 \mathrm{H}$ mice primed by injection of various DCs all exhibited significant low proliferative response to syngeneic $\mathrm{Ag}(\mathrm{C} 3 \mathrm{H})$ pulsed DCs.

3.3. DCs after CD80 and CD86 Blockade Prolongs Heart Allograft Survival. To investigate the therapeutic potential of these equipped DCs in murine cardiac transplant model, recipient mice $(\mathrm{C} 3 \mathrm{H})$ were pretreated with $2 \times 10^{6}$ DCs in $N C$ lenti group, or CD80 lenti and CD86 lenti group, 7 days before heterotopic heart transplantation from $\mathrm{B} 6$ to $\mathrm{C} 3 \mathrm{H}$. Control group was pretreated with PBS. Median survival time (MST) in the control group was 9.3 days, while NC lenti-transduced DCs pretreatment accelerated heart allograft rejection (MST 6 days compared to control group). Pretreatment with CD80 lenti and CD86 lenti-transduced DCs significantly prolonged the MST to 21.8 days but had no effect on pulsing third party Ag (MST 7.5 days). These findings suggest that infusion of CD80 lenti and CD86 lenti-transduced DCs-pulsed alloAg prior to transplantation prolongs heart allograft survival, and the prolongation is Ag specific (Figure 5).

3.4. CD80 Lenti and CD86 Lenti-Transduced DCs Increase T Cell Apoptosis. The most striking finding from the current study was the possible mechanism of equipped DCs prolonging graft survival. Animals were sacrificed 5 days after transplantation. Lymphocytes were isolated from spleens, mesenteric lymph nodes, and grafts and were assessed by Annexin $\mathrm{V}$ staining. Apoptotic cell in $\mathrm{CD} 3+\mathrm{T}$ cell population from mice receiving CD80 lenti and CD86 lentitransduced DCs was markedly higher than the T cells in NC lenti group (Figure 6).

3.5. ERS-Mediated Apoptosis Pathway Is Involved in Allogeneic T-Cell Apoptosis. To better characterize the mechanism of $\mathrm{T}$ cell apoptosis after costimulatory blockade, we used RTPCR to analyze a number of apoptosis signaling pathway molecules in recipient spleens. As shown in Figure 7, a striking upregulation of Bax and GRP78 was found in recipients treated with CD80 lenti and CD86 lenti-transduced DCs compared to NClenti-transduced DCs or PBS. These changes in endoplasmic reticulum and mitochondrial pathway were enhanced by upregulation of CHOP and suppression of Bcl$\mathrm{xL}$ expression in these cells. But there were no remarkable changes in death receptor pathway among three groups.

\section{Discussion}

The success of organ transplantation depends on the continuous administration of nonspecific immunosuppressive agents, immunosuppression therapies present risks for opportunistic infection, malignancy, and a variety of agent side effects [21]. Progress has recently been made in dissecting the APC and T cell interactions. In particular, several DC-based therapies in blocking the costimulation pathways have achieved encouraging results [3-8]. However, antibodies or fusion proteins have a limited half-life and require multiple antibody treatments [22]. High cost and toxicity restrict the application of the antisense technology [23]. Adenoviruses are efficient transducers but they activate immune responses [24]. Therefore we now apply RNAi technique to equipped DCs. Our previous studies have indicated that chemically synthesized siRNA can specifically and effectively knock down CD80 and CD86 gene expression in BM-derived DCs [25]. Inhibition of short duration potentially limits their use in vivo. Then we choose recombinant lentiviruses that contain stem-loop constructs encoding that hairpin RNAs lend to the intracellular generation of siRNA-like species [26]. Lentivirus can infect noncycling and postmitotic cells; reverse transcribed DNA sequences can subsequently integrate into the genome of target cells and are stably expressed at high levels for extended times [27].

Our data clearly demonstrate that lentivirus transduced DCs induce $\mathrm{T}$ cell hyporesponsiveness and T cells primed by alloAg-pulsed DCs later inhibit allogeneic responses, in an Ag-specific manner. The findings are similar with Taner et al. [10] who showed that T cells cross-primed by alloAg-pulsed, rapamycin treated DCs were hyporesponsive to subsequent challenge. Recently, a third pathway called semi-direct pathway has been proposed. Recipient DCs can acquire intact MHC molecules from donor cells or tissues and stimulate direct antidonor alloimmune responses [28]. One study by Mandelbrot et al. [29] showed that MHC-II-/-CD4+ recipients could reject CD80-/-CD86-/- cardiac grafts as rapidly as wild-type grafts. The observed acute graft rejection may have been due to the direct activation of recipient $\mathrm{T}$ cells by recipient APCs after acquiring intact donor MHC 

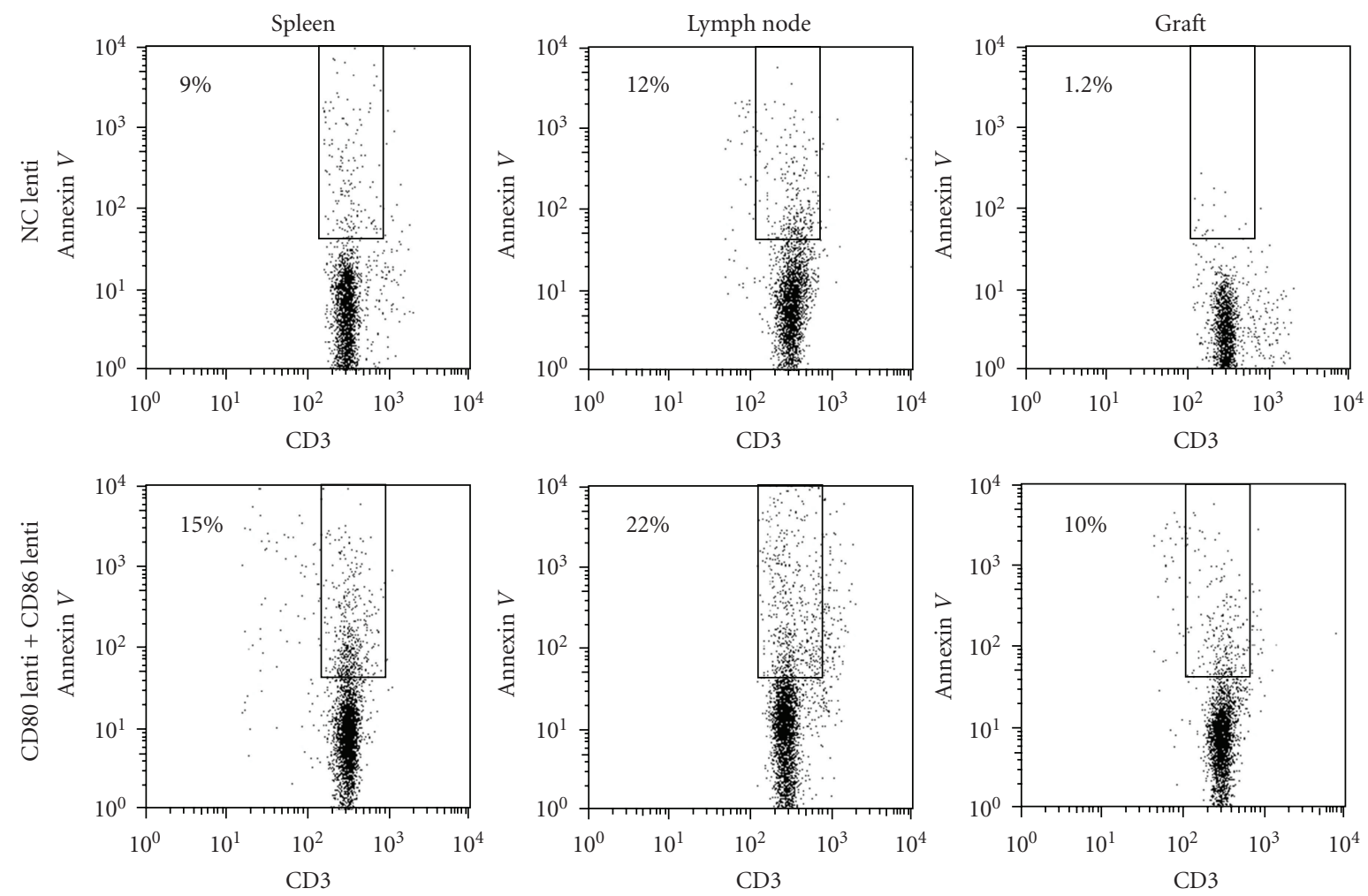

FIGURE 6: Lymphocytes were isolated from recipient spleens, mesenteric lymph nodes, and grafts on day 5 after transplantation treated with recombinant lentivirus transduced DCs; these cells were double stained with FITC-conjugated anti-CD3 and PE-conjugated Annexin V, analyzed by flow cytometry. Numbers indicate the percentage of Annexin V positive cells in CD3+ cell population.

molecules. These showed that recipient DCs appeared to be a key driver of graft dysfunction. The induction of transplant tolerance has therefore to focus on these cells.

The CD80/CD86-CD28 pathway is probably the most important and best characterized in T cell activation [30]; we have shown herein that blockade of CD80 and CD86 by lentivirus mediated RNAi increases cardiac allograft survival but does not extend it indefinitely. This result indicates that other costimulatory pathways may regulate allogeneic immune responses. Since co-stimulation blockade is less effective in memory $\mathrm{T}$ cells than in naive $\mathrm{T}$ cells [31], memory $\mathrm{T}$ cells may also play an active role. There are additional factors to deliberate in DC immunotherapy. Several groups have addressed whether one or multiple injections will achieve a more significant effect. Further, the optimal number of DCs to be injected needs to be addressed. Also, the DCs timing strategies will have to be considered.

The mechanism by which lentivirus transduced DCs prolong heart allograft survival remains to be determined. The key requirement for long-term survival is that none of the activated effector $\mathrm{T}$ cells survives in a functional state [32]. There are three distinct pathways of $\mathrm{T}$ cell apoptosis, which may be interconnected. These include death receptor pathway, mitochondrial pathway and most recently described the endoplasmic reticulum pathway [33]. The first pathway occurs in repetitively stimulated $\mathrm{T}$ cells and is largely mediated through Fas and through related members of the TNF-receptor family. The second pathway occurs when activated $\mathrm{T}$ cells are deprived of growth factors. Bcl-2 family proteins control the release of cytochrome $\mathrm{c}$ and apoptosis-inducing factor from mitochondria [34]. Sen's group has found that alloreactive $\mathrm{T}$ cell apoptosis is induced via Fas/FasL pathway in cardiac allograft $[35,36]$. Wekerle et al. [37] showed that the peripheral deletion of donorreactive $\mathrm{T}$ cells in the early period after $\mathrm{BM}$ transplantation with costimulatory blockade using anti-CD154 and CTLA4Ig was mediated in part by Fas and could be overcome by the constitutive expression of Bcl-xL. Under ERS, unfolded proteins accumulate in the lumen of the endoplasmic reticulum, and then cells activate a self-protective mechanism by activation of genes encoding endoplasmic reticulumresident chaperones such as BiP/GRP78 and GRP94. If these adaptive responses are not sufficient to relieve cells from ERS, cells undergo apoptosis associated with several molecules, including CHOP, caspase-12, and MAP kinase cascade [38]. Several investigators have reported that ERS is involved in B cell differentiation and the innate immune response [39]. ERS response influences ischemia-reperfusion in human liver transplantation [40], while the endoplasmic reticulum pathway-mediated apoptosis in $\mathrm{T}$ cells of the immune system has not been studied. First we observed that, in recipient spleens, GRP78 and CHOP were much higher in recombinant lentivirus group. Furthermore, $\mathrm{T}$ cells induced to undergo apoptosis can release IL-10 as they die. Phagocytosis of apoptotic cells by macrophages often leads to the production of IL-10 and TGF and may promote 


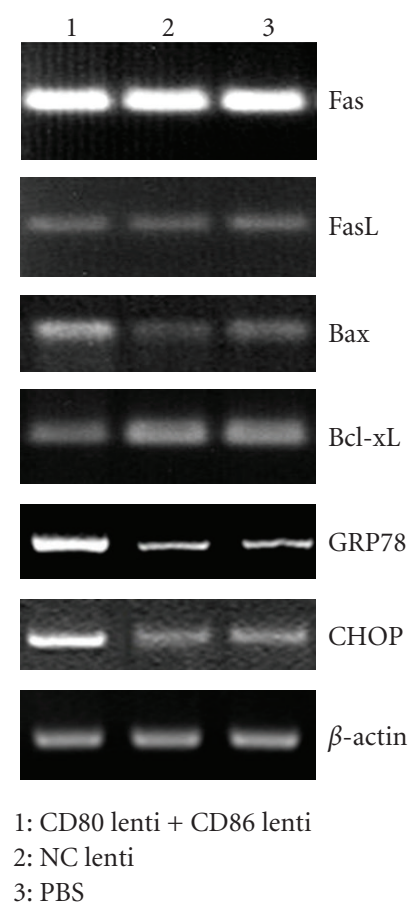

FIGURE 7: T cells isolated on day 5 after translation from the spleens of $\mathrm{C} 3 \mathrm{H}$ recipients bearing $\mathrm{B} 6$ heart allografts treated with $\mathrm{PBS}$ or recombinant lentivirus transduced DCs were used for analysis of T cell apoptosis signaling pathway molecules by RT-PCR. All data are representative of three separate experiments.

tolerance [34]. Nevertheless, it is conceivable that apoptosis of activated $\mathrm{T}$ cells not only reduces the mass of $\mathrm{T}$ cells but also promotes active immune regulation that is essential for long-lasting tolerance.

In summary, RNAi-treated, alloAg-pulsed host DCs can induce Ag-specific $\mathrm{T}$ cell hyporesponsiveness and prolong heart allograft survival. Apoptosis of alloreactive $\mathrm{T}$ cells is critical for induction of stable peripheral allograft tolerance. ERS-mediated apoptosis pathway may play a role in peripheral tolerance after heart transplantation with costimulatory blockade. A detailed understanding of activated $\mathrm{T}$ cells apoptosis in this process will undoubtedly lead to the design of more effective strategies to achieve tolerance in the clinic.

\section{Acknowledgments}

Xiaodong Gu and Jianbin Xiang have equally contributed to this work. They are joint first authors. The authors thank Shanghai GeneChem Co., Ltd for technical support and valuable discussion. Xiaodong $\mathrm{Gu}$ and Jianbin Xiang have equally contributed to this work. A grant from National Natural Science Foundation of China (no. 30772035), a grant from Interdisciplinary Research Fund for Basic-Clinical Medicine of Shanghai Medical College at Fudan University, an open fund from Institutes of Biomedical Sciences at Fudan University, a grant from "Star of Century" Foundation of Fudan University, a grant from Reserve Talents Training Plan for Shanghai Excellent Young Teachers and a grant from Natural Science Foundation of Shanghai (no. 09ZR1405200).

\section{References}

[1] A. E. Morelli and A. W. Thomson, "Dendritic cells: regulators of alloimmunity and opportunities for tolerance induction," Immunological Reviews, vol. 196, pp. 125-146, 2003.

[2] R. H. Schwartz, "T cell anergy," Annual Review of Immunology, vol. 21, pp. 305-334, 2003.

[3] N. Giannoukakis, C. A. Bonham, S. Qian, et al., "Prolongation of cardiac allograft survival using dendritic cells treated with NF- $\kappa$ B decoy oligodeoxyribonucleotides," Molecular Therapy, vol. 1, no. 5, pp. 430-437, 2000.

[4] C. A. Bonham, L. Peng, X. Liang, et al., "Marked prolongation of cardiac allograft survival by dendritic cells genetically engineered with NF- $\kappa \mathrm{B}$ oligodeoxyribonucleotide decoys and adenoviral vectors encoding CTLA4-Ig," Journal of Immunology, vol. 169, no. 6, pp. 3382-3391, 2002.

[5] X. Liang, L. Lu, Z. Chen, et al., "Administration of dendritic cells transduced with antisense oligodeoxyribonucleotides targeting CD80 or CD86 prolongs allograft survival," Transplantation, vol. 76, no. 4, pp. 721-729, 2003.

[6] Y. Kita, X.-K. Li, M. Ohba, et al., "Prolonged cardiac allograft survival in rats systemically injected adenoviral vectors containing CTLA4Ig-gene," Transplantation, vol. 68, no. 6, pp. 758-766, 1999.

[7] S. Tomasoni, S. Aiello, L. Cassis, et al., "Dendritic cells genetically engineered with adenoviral vector encoding dnIKK2 induce the formation of potent CD4+ T-regulatory cells," Transplantation, vol. 79, no. 9, pp. 1056-1061, 2005.

[8] F. Vincenti, C. Larsen, A. Durrbach, et al., "Costimulation blockade with belatacept in renal transplantation," New England Journal of Medicine, vol. 353, no. 8, pp. 770-781, 2005.

[9] N. J. Rogers and R. I. Lechler, "Allorecognition," American Journal of Transplantation, vol. 1, no. 2, pp. 97-102, 2001.

[10] T. Taner, H. Hackstein, Z. Wang, A. E. Morelli, and A. W. Thomson, "Rapamycin-treated, alloantigen-pulsed host dendritic cells induce Ag-specific T cell regulation and prolong graft survival," American Journal of Transplantation, vol. 5, no. 2, pp. 228-236, 2005.

[11] R. Lechler, W. F. Ng, and R. M. Steinman, "Dendritic cells in transplantation-friend or foe?" Immunity, vol. 14, no. 4, pp. 357-368, 2001.

[12] D. A. Mandelbrot, Y. Furukawa, A. J. McAdam, et al., "Expression of $\mathrm{B} 7$ molecules in recipient, not donor, mice determines the survival of cardiac allografts," Journal of Immunology, vol. 163, no. 7, pp. 3753-3757, 1999.

[13] D.-L. Xu, Y. Liu, J.-M. Tan, et al., "Marked prolongation of murine cardiac allograft survival using recipient immature dendritic cells loaded with donor-derived apoptotic cells," Scandinavian Journal of Immunology, vol. 59, no. 6, pp. 536544, 2004.

[14] M.-M. Tiao, L. Lu, R. Tao, L. Wang, J. J. Fung, and S. Qian, "Prolongation of cardiac allograft survival by systemic administration of immature recipient dendritic cells deficient in NF- $\kappa$ B activity," Annals of Surgery, vol. 241, no. 3, pp. 497505, 2005.

[15] H. Auchincloss Jr., R. Lee, S. Shea, J. S. Markowitz, M. J. Grusby, and L. H. Glimcher, "The role of 'indirect' recognition in initiating rejection of skin grafts from major histocompatibility complex class II-deficient mice," Proceedings of the National Academy of Sciences of the United States of America, vol. 90, no. 8, pp. 3373-3377, 1993. 
[16] J. A. Hill, T. E. Ichim, K. P. Kusznieruk, et al., "Immune modulation by silencing IL-12 production in dendritic cells using small interfering RNA," Journal of Immunology, vol. 171, no. 2, pp. 691-696, 2003.

[17] H. Kadowaki, H. Nishitoh, and H. Ichijo, "Survival and apoptosis signals in ER stress: the role of protein kinases," Journal of Chemical Neuroanatomy, vol. 28, no. 1-2, pp. 93100, 2004.

[18] N. Pallet, S. Fougeray, P. Beaune, C. Legendre, E. Thervet, and D. Anglicheau, "Endoplasmic reticulum stress: an unrecognized actor in solid organ transplantation," Transplantation, vol. 88, no. 5, pp. 605-613, 2009.

[19] M. B. Lutz, N. Kukutsch, A. L. J. Ogilvie, et al., "An advanced culture method for generating large quantities of highly pure dendritic cells from mouse bone marrow," Journal of Immunological Methods, vol. 223, no. 1, pp. 77-92, 1999.

[20] D. A. Rubinson, C. P. Dillon, A. V. Kwiatkowski, et al., "A lentivirus-based system to functionally silence genes in primary mammalian cells, stem cells and transgenic mice by RNA interference," Nature Genetics, vol. 33, no. 3, pp. 401406, 2003.

[21] S. Jiang, O. Herrera, and R. I. Lechler, "New spectrum of allorecognition pathways: implications for graft rejection and transplantation tolerance," Current Opinion in Immunology, vol. 16, no. 5, pp. 550-557, 2004.

[22] I. W. Flinn and H. M. Lazarus, "Monoclonal antibodies and autologous stem cell transplantation for lymphoma," Bone Marrow Transplantation, vol. 27, no. 6, pp. 565-569, 2001.

[23] N. Dias and C. A. Stein, "Antisense oligonucleotides: basic concepts and mechanisms.", Molecular Cancer Therapeutics, vol. 1, no. 5, pp. 347-355, 2002.

[24] E. Marshall, "Gene therapy death prompts review of adenovirus vector," Science, vol. 286, no. 5448, pp. 2244-2245, 1999.

[25] X. Gu, J. Xiang, Y. Yao, and Z. Chen, "Effects of RNA interference on CD80 and CD86 expression in bone marrow-derived murine dendritic cells," Scandinavian Journal of Immunology, vol. 64, no. 6, pp. 588-594, 2006.

[26] S. A. Stewart, D. M. Dykxhoorn, D. Palliser, et al., "Lentivirusdelivered stable gene silencing by RNAi in primary cells," RNA, vol. 9, no. 4, pp. 493-501, 2003.

[27] G. Perletti, D. Osti, E. Marras, G. Tettamanti, and M. De Eguileor, "Generation of VSV-G pseudotyped lentiviral particles in 293T cells," Journal of Cellular and Molecular Medicine, vol. 8, no. 1, pp. 142-143, 2004.

[28] A. Caballero, N. Fernandez, R. Lavado, M. J. Bravo, J. M. Miranda, and A. Alonso, "Tolerogenic response: allorecognition pathways," Transplant Immunology, vol. 17, no. 1, pp. 3-6, 2006.

[29] D. A. Mandelbrot, K. Kishimoto, H. Auchincloss Jr., A. H. Sharpe, and M. H. Sayegh, "Rejection of mouse cardiac allografts by costimulation in trans," Journal of Immunology, vol. 167, no. 3, pp. 1174-1178, 2001.

[30] R. Snanoudj, H. De Préneuf, C. Créput, et al., "Costimulation blockade and its possible future use in clinical transplantation," Transplant International, vol. 19, no. 9, pp. 693-704, 2006.

[31] A. Valujskikh, B. Pantenburg, and P. S. Heeger, "Primed allospecific T cells prevent the effects of costimulatory blockade on prolonged cardiac allograft survival in mice," American Journal of Transplantation, vol. 2, no. 6, pp. 501-509, 2002.

[32] N. J. Monk, R. E. G. Hargreaves, E. Simpson, J. P. Dyson, and S. Jurcevic, "Transplant tolerance: models, concepts and facts," Journal of Molecular Medicine, vol. 84, no. 4, pp. 295 304,2006
[33] S. Gupta and S. Gollapudi, "Molecular mechanisms of TNF$\alpha$-induced apoptosis in naïve and memory T cell subsets," Autoimmunity Reviews, vol. 5, no. 4, pp. 264-268, 2006.

[34] X. C. Li, A. D. Wells, T. B. Strom, and L. A. Turka, "The role of T cell apoptosis in transplantation tolerance," Current Opinion in Immunology, vol. 12, no. 5, pp. 522-527, 2000.

[35] T. C. Tung, K. Oshima, G. Cui, H. Laks, and L. Sen, "Dual upregulation of Fas and Bax promotes alloreactive T cell apoptosis in IL-10 gene targeting of cardiac allografts," American Journal of Physiology, vol. 285, no. 3 54-3, pp. H964H973, 2003.

[36] H. Furukawa, K. Oshima, T. Tung, G. Cui, H. Laks, and L. Sen, "Overexpressed exogenous IL-4 and IL-10 paradoxically regulate allogenic $\mathrm{T}$-Cell and cardiac myocytes apoptosis through FAS/FASL pathway," Transplantation, vol. 85, no. 3, pp. 437-446, 2008.

[37] T. Wekerle, J. Kurtz, M. H. Sayegh, et al., "Peripheral deletion after bone marrow transplantation with costimulatory blockade has features of both activation-induced cell death and passive cell death," Journal of Immunology, vol. 166, no. 4, pp. 2311-2316, 2001.

[38] H. Kadowaki, H. Nishitoh, and H. Ichijo, "Survival and apoptosis signals in ER stress: the role of protein kinases," Journal of Chemical Neuroanatomy, vol. 28, no. 1-2, pp. 93100, 2004.

[39] L. Zhao and S. L. Ackerman, "Endoplasmic reticulum stress in health and disease," Current Opinion in Cell Biology, vol. 18, no. 4, pp. 444-452, 2006.

[40] A. Emadali, D. T. Nguyên, C. Rochon, G. N. Tzimas, P. P. Metrakos, and E. Chevet, "Distinct endoplasmic reticulum stress responses are triggered during human liver transplantation," Journal of Pathology, vol. 207, no. 1, pp. 111-118, 2005. 


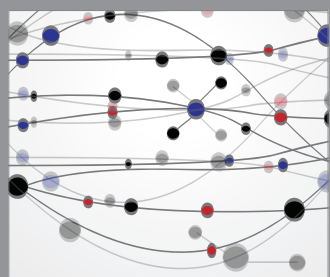

The Scientific World Journal
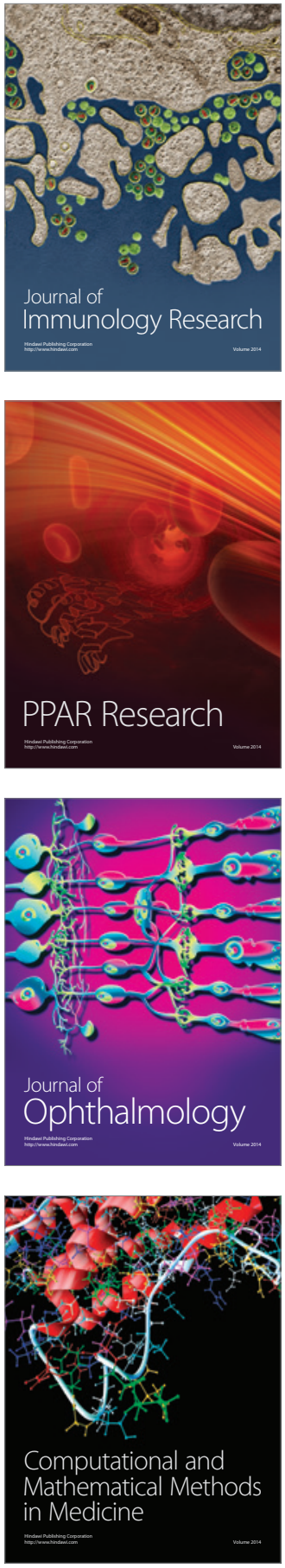

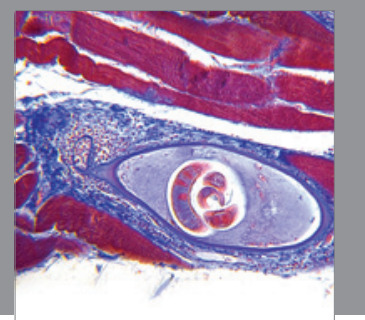

Gastroenterology

Research and Practice
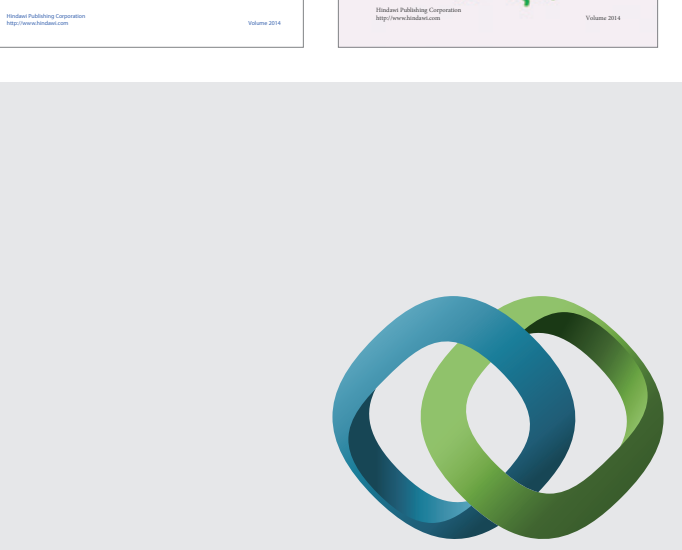

\section{Hindawi}

Submit your manuscripts at

http://www.hindawi.com
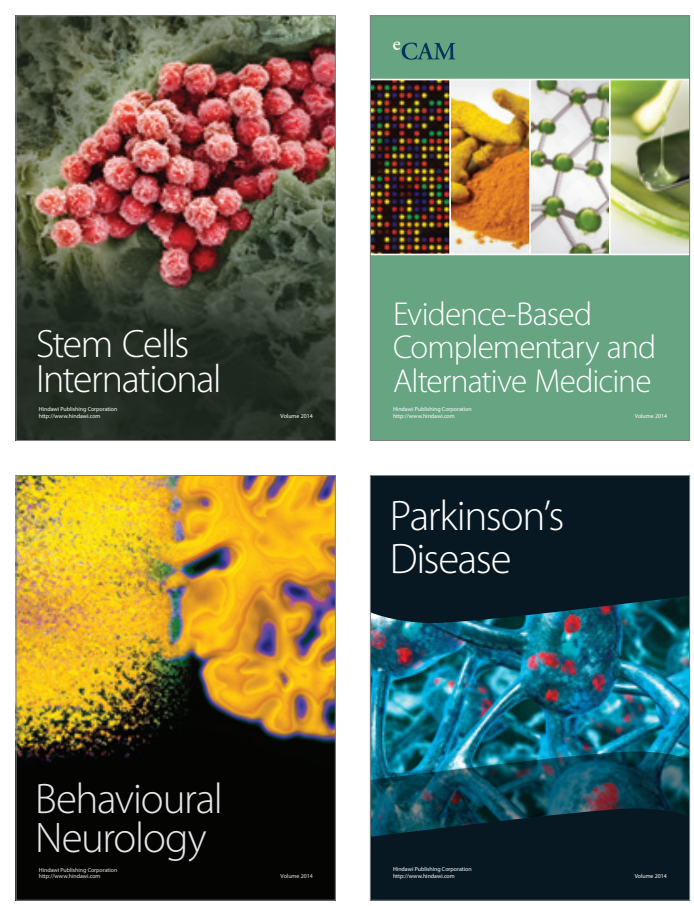

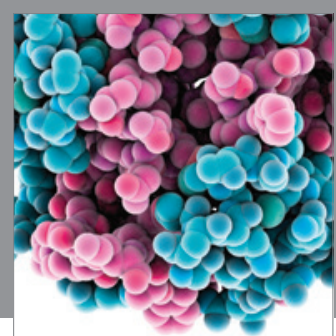

Journal of
Diabetes Research

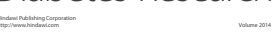

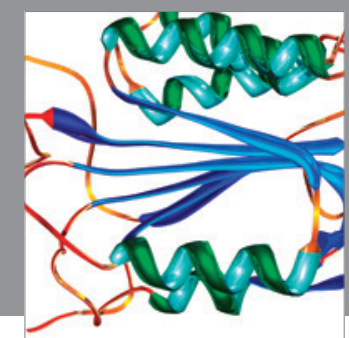

Disease Markers
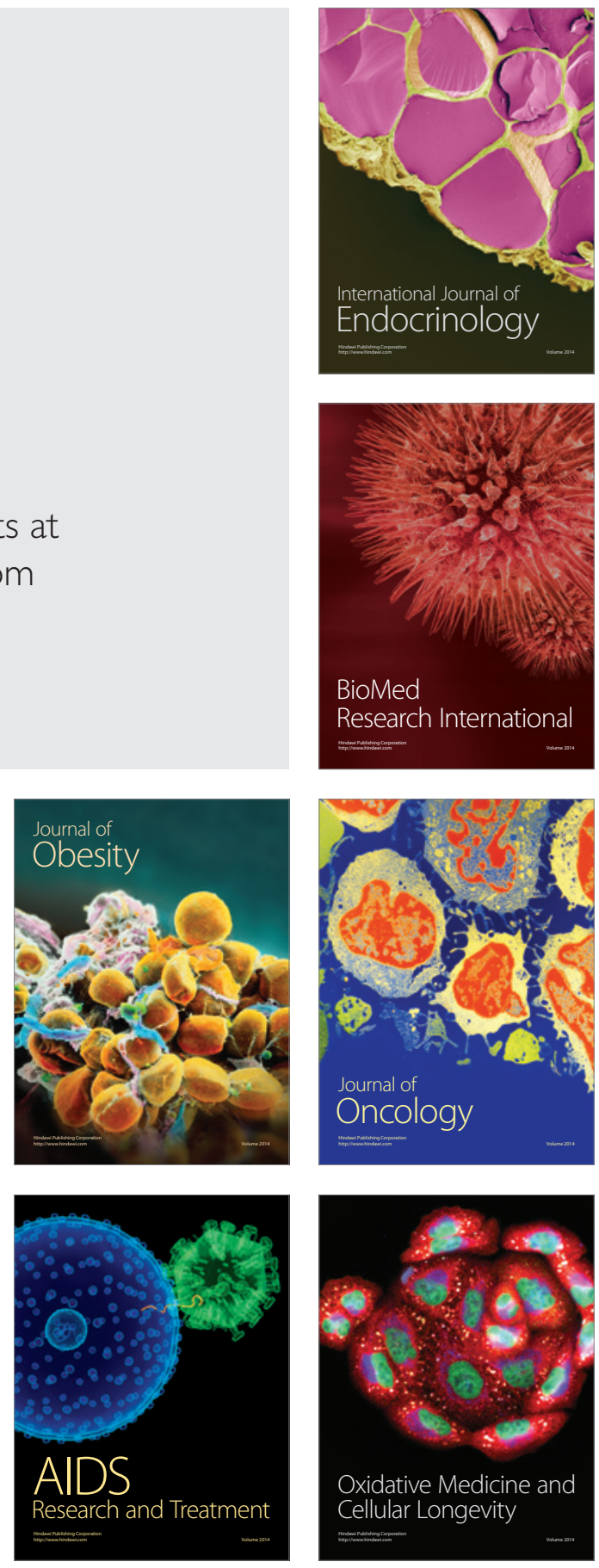